\title{
Detection of Malicious Intent in Non-cooperative Drone Surveillance
}

\author{
Jiaming Liang ${ }^{\dagger}$, Bashar I. Ahmad ${ }^{\ddagger}$, Mohammad Jahangir ${ }^{\S}$ and Simon Godsill ${ }^{\dagger}$ \\ ${ }^{\dagger}$ Engineering Department, University of Cambridge, UK \\ ${ }^{\ddagger}$ Aveillant, Thales Land and Air Systems, Cambridge, UK \\ $\S$ Department of Electronic, Electrical and Systems Engineering, University of Birmingham, UK \\ Emails: j1809@cam.ac.uk bashar.ahmad@aveillant.com m.jahangir@bham.ac.uk sjg30@cam.ac.uk
}

\begin{abstract}
In this paper, a Bayesian approach is proposed for the early detection of a drone threatening or anomalous behaviour in a surveyed region. This is in relation to revealing, as early as possible, the drone intent to either leave a geographical area where it is authorised to fly (e.g. to conduct inspection work) or reach a prohibited zone (e.g. runway protection zones at airports or a critical infrastructure site). The inference here is based on the noisy sensory observations of the target state from a non-cooperative surveillance system such as a radar. Data from Aveillant's Gamekeeper radar from a live drone trial is used to illustrate the efficacy of the introduced approach.

Index Terms-Bayesian inference, drone, intent prediction, Kalman filtering, non-cooperative surveillance, radar
\end{abstract}

\section{INTRODUCTION}

Small unmanned air systems (sUASs) or drones are poised to play an increasingly important role in various fields given their high potential benefits, for example in agriculture, ecommerce, media, inspection and maintenance, to name a few. The trends in UAS technologies suggest that the low altitude airspace could be populated by different drone platforms in the near future. This motivated large initiatives such as the EU Single European Sky ATM Research (SESAR) Joint Undertaking programme [1] to enable complex drone operations with a high degree of automation. This encompasses ground-based technologies for real-time Unmanned Traffic Management System (UTMS). However, the threat drones can pose to the safety and security of the public (e.g. operating in the presence of manned aviation) is widely recognised and non-cooperative drone surveillance is hence key for UMTS.

Several civilian ground UAS surveillance technologies have emerged, where typically only radar sensors can detect sUASs at long distances and wide ranges [2]-[4], unlike for example electro-optical/infrared and acoustic ones. Nonetheless, they often lack the means to determine which of the detected drones require the operator's (urgent) attention or further scrutiny or even necessitate triggering an alarm (e.g. closing the airspace). This is particularly critical in the future as scenes become more congested with the commercial use of sUAS and UTMS operators can be easily overwhelmed.

In this paper, we attempt to address from a Bayesian perspective the problem of predicting, as early as possible,

This work is funded by the Defence Science and Technology Laboratory (DSTL) under the DASA contract DSTLX1000144447. anomalous or threatening behaviour of a target based on noisy sensory observations, for instance from a radar. Anomaly or threat here is defined as the UAS intending to leave (maliciously or not) a geographic region which it is authorised to fly in (e.g. to carry out surveillance or inspection tasks) or reach prohibited area(s) such as no-fly zones near airports [5]. Real radar data is used to demonstrate the performance of the proposed approach. Such predictive capability can be crucial for smart UMTS solutions because it facilitates automated decision making and prioritisation of potential threats for effective resources allocation, e.g. operator's attention or secondary systems. It can also circumvent the need for human-intensive inspection and provide sufficient time to avoid triggering drastic measures (e.g. warning a hobbyist drone operator instead of closing the airspace).

\section{A. Problem Formulation}

Let $\mathcal{A}$ be the authorised flying zone for a sUAS and $\mathbf{m}_{n}$ at time instant $t_{n}$ be the drone state (e.g. its location in 3$\mathrm{D}$ or any other spatio-temporal characteristics) measured by a non-cooperative surveillance sensor (e.g. radar). The objective in this paper is to sequentially estimate the probability, $\operatorname{Pr}\left(\right.$ Des $\left.=\mathcal{A} \mid \mathbf{m}_{0: n}\right)$, of $\mathcal{A}$ being the intended destination of the UAS, i.e. the drone intends to remain in this area. The observation sequence $\mathbf{m}_{0: n}$ is made successively at ordered time instants $\left\{t_{0}, t_{1}, \ldots, t_{n}\right\}$. Similarly, $\mathcal{A}$ can be defined as a prohibited flying region for the UAS and the task becomes monitoring the probability of the drone aiming to reach it. With the proposed intent inference approach a number of areas, $\mathcal{A}_{i}, i=1,2, \ldots, M$, can be simultaneously considered and no restriction on the region(s) shape is imposed in principle.

\section{B. Related Work and Contributions}

In recent years there has been a surging interest in predicting intent (such as destination or future actions) of tracked objects owing to its potential applications in smart navigation, robotics, etc. Some methods, dubbed data-driven for instance [6]-[8], capitalise on the availability of sufficiently large data to train a prediction model (e.g. a neural network). However, such training data may not be always available and its collection-labelling can be prohibitively expensive. For instance, data of drones flying within a surveillance area is often limited due to operational constraints. Therefore, the 
intent prediction problem in this paper is tackled within a Bayesian object tracking framework with minimal training data requirements, only to parameterise the underlying models. A model-based approach is subsequently designed which can effectively infer the un-observable UAS intent.

Existing model-based intent inference techniques in object tracking are largely focused on: 1) determining the target final destination out of a finite set of nominal endpoints at known locations, e.g. [9]-[13], see [14], [15] for an overview; 2) destination-aware tracking based on reciprocal processes [16] for pre-defined endpoints; 3) long-term trajectory forecasting using motion models learnt in advance, e.g. [17], [18]. However, they either cannot be directly applied to scenarios where the object destination is unknown a priori, time-varying and can be anywhere in the considered region (i.e. a continuous intent space), e.g. [9]-[15], or require an off-line training phase for the learning of intent parameters [17], [18].

Compared to the above related work, here we introduce a more natural solution to the considered intent prediction problem which relies on devising a dynamical model to the latent intent and connecting it to the target (e.g. UAS) kinematic states via some carefully designed stochastic process. The idea is close in spirit to the virtual leader model [19], [20] and the bulk model [21] for group tracking and has recently been used for destination inference in [22], [23]. More specifically, in this paper we

- introduce a probabilistic framework for the early detection of threatening/malicious drone intent (e.g. exiting or reaching $\mathcal{A}$ ) in non-cooperative surveillance contexts and demonstrate its performance using real radar data,

- adopt a new modelling approach based on the virtual leader setting in [20], however not for group object tracking, but for predicting the target intent. The latter can dynamically change over time here and the destination sought by the object/UAS can be an extended spatial region of any shape, located anywhere in the surveyed area. Thus, it does not require the prior knowledge of a set of possible endpoints as in [9]-[15],

- propose a simple, low-complexity, Kalman filter-based inference scheme based on continuous state-space models and asynchronous measurements can be treated.

\section{Motion And InTEnT Model}

Since the movement pattern of a target is often related to its underlying intent (e.g. reaching or remaining in a given location or area), we consider a dynamical model that represents the object motion under the influence of a latent destination. Here, this hidden destination can dynamically change over time and it is continuous in space. Specifically, the applied model has the following state transition density

$$
p\left(\mathbf{s}_{n+1} \mid \mathbf{s}_{n}\right)=p\left(\mathbf{x}_{n+1}, \mathbf{r}_{n+1} \mid \mathbf{x}_{n}, \mathbf{r}_{n}\right)
$$

with $\mathbf{s}_{n}=\left[\mathbf{x}_{n}, \mathbf{r}_{n}\right]^{T}$ being the overall state variable at $t_{n}$. Here, $\mathbf{x}_{n}$ consists of object kinematic states such as position, velocity and acceleration while $\mathbf{r}_{n}$ is a latent variable for the target/drone intent. Models with the structure depicted by (1) have been widely studied and appeared under various guises in the object tracking field. Examples include the virtual leader model, e.g. [19], [20], the bulk model [21], and the latent destination model [23]. In this paper, we adopt the following linear stochastic differential equations (SDEs) for the object motion $\mathbf{x}_{t}=\left[x_{t}, \dot{x}_{t}\right]^{T}$ and the intended destination location $\mathbf{r}_{t}=r_{t}^{x}$ in a 1-dimensional Cartesian coordinate system

$$
\begin{aligned}
d \dot{x}_{t} & =\left\{\eta_{x}\left(r_{t}^{x}-x_{t}\right)-\rho_{x} \dot{x}_{t}\right\} d t+\sigma_{x} d B_{t} \\
d r_{t}^{x} & =\sigma_{r} d B_{t}
\end{aligned}
$$

with $\eta_{x}$ and $\rho_{x}$ being positive constants. $B_{t}$ is a 1-dimensional standard Brownian motion and $\sigma_{x}, \sigma_{r_{x}}$ are the diffusion constants. According to (2), the position and velocity of the object will revert towards the location of the latent destination at $r_{t}^{x}$ and 0 , respectively. The zero-reverting part $-\rho_{x} \dot{x}_{t}$ serves to prevent the object velocity from becoming excessively high. Consequently, (1) can be factorised as

$$
p\left(\mathbf{s}_{n+1} \mid \mathbf{s}_{n}\right)=p\left(\mathbf{x}_{n+1} \mid \mathbf{x}_{n}, \mathbf{r}_{n}\right) p\left(\mathbf{r}_{n+1} \mid \mathbf{r}_{n}\right)
$$

This model can be readily adapted to scenarios where the object has a fixed final destination by setting $\sigma_{r}=0$. It is also possible to include higher-order kinematics, for instances, $\mathbf{s}_{t}=\left[x_{t}, \dot{x}_{t}, r_{t}^{x}, \dot{r}_{t}^{x}\right]^{T}$ with the velocity $\dot{r}_{t}^{x}$ the object velocity reverts to being part of the state. In this case, the model bears a resemblance to the group virtual leader model [20] with the number of objects being one. Moreover, the presented model can be used for estimating the intended destination of a fastmanoeuvring object if (2) is, for example, Lévy process-driven [23]. The above SDEs can be formalised in a vector-matrix form as below, with $\mathbf{s}_{t}=\left[x_{t}, \dot{x}_{t}, r_{t}^{x}\right]^{T}$,

$$
d \mathbf{s}_{t}=A \mathbf{s}_{t} d t+L d B_{t}^{s}
$$

$$
A=\left[\begin{array}{ccc}
0 & 1 & 0 \\
-\eta_{x} & -\rho_{x} & \eta_{x} \\
0 & 0 & 0
\end{array}\right], L=\left[\begin{array}{cc}
0 & 0 \\
\sigma_{x} & 0 \\
0 & \sigma_{r}
\end{array}\right], B_{t}^{s}=\left[B_{t}, B_{t}\right]^{T}
$$

A $k$-dimensional system model can be obtained readily by cascading several such 1-dimensional models. It can be shown that the solution to (4) is given by:

$$
\mathbf{s}_{n}=F_{\tau} \mathbf{s}_{n-1}+\int_{0}^{\tau} e^{A(\tau-u)} L d B_{u}^{s}
$$

with $\tau=t_{n}-t_{n-1}$ and $F_{\tau}=e^{A \tau}$. Correspondingly, the state transition density is of a Gaussian form:

$$
p\left(\mathbf{s}_{n} \mid \mathbf{s}_{n-1}\right)=\mathcal{N}\left(\mathbf{s}_{n} \mid F_{\tau} \mathbf{s}_{n-1}, Q_{\tau}\right)
$$

where $Q_{\tau}=\int_{0}^{\tau} e^{A(\tau-u)} L L^{T} e^{A(\tau-u)^{T}} d u$.

It is emphasised that with this modelling approach the target destination can: a) dynamically change over time subject to the underlying stochastic model; b) be anywhere within the surveyed region since $\mathbf{r}_{n}$ is a continuous random variable with a support $\mathbb{R}^{k}$, where $k=1,2$ or 3 .

\section{Proposed Intent Prediction Method}

The pseudo-code of the proposed, simple, intent inference routine at time $t_{n}$ is provided in Algorithm 1. Next, we detail how this predictor is formulated. 


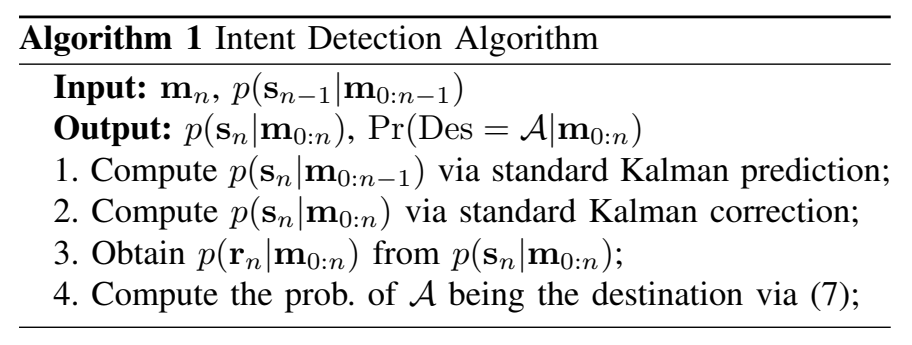

\section{A. State estimation}

The aim here is to estimate the latent destination density $p\left(\mathbf{r}_{n} \mid \mathbf{m}_{0: n}\right)$ given indirect sensor observations made up to the current time $t_{n}$, for instance noisy measurements of a target trajectory in 2-D or 3-D. This can be the drone location produced by the Gamekeeper radar as in Section IV. The observation density is given by

$$
p\left(\mathbf{m}_{n} \mid \mathbf{s}_{n}\right)=\mathcal{N}\left(\mathbf{m}_{n} \mid G_{n} \mathbf{s}_{n}, \Sigma_{n}\right)
$$

where the observation matrix $G_{n}$ extracts the Cartesian coordinates from the state vector while $\Sigma_{n}$ is the noise covariance matrix. This particular setting immediately permits us to use a Kalman filter for the online state estimation task. As a result, we can easily obtain the desired latent destination posterior density $p\left(\mathbf{r}_{n} \mid \mathbf{m}_{0: n}\right)$ as a marginal of the overall Gaussian distribution $p\left(\mathbf{s}_{n} \mid \mathbf{m}_{0: n}\right)$. Nonetheless, it is worth pointing out that the method proposed here is not limited to linear Gaussian systems. When non-linear and/or non-Gaussian observation model is treated, a particle filtering based scheme can be applied for state (including intent) estimation.

\section{B. Spatial region as the intended destination}

Since the posterior density of intent is estimated in this paper as described above, the probability that a target aims to leave (or reach) an extended spatial region (or any point within it) can be readily obtained by evaluating the integral

$$
\operatorname{Pr}\left(\text { Des }=\mathcal{A} \mid \mathbf{m}_{0: n}\right)=\int_{\mathcal{A}} p\left(\mathbf{r}_{n} \mid \mathbf{m}_{0: n}\right) d \mathbf{r}_{n},
$$

where $\mathcal{A}$ is the authorised/prohibited region. The integral in (7) can be easily calculated for a Gaussian (or a mixture of Gaussians) and regions of rectangular (or cuboid) shapes can simplify the evaluation (e.g. using the multivariate cumulative distribution functions). For other distributions and geometric shapes, approximations can be applied and tractability of the integral estimation should be considered. Whilst this will be treated in more detail in future work, it is not expected to lead to drastically different outcomes to approximating the area of interest with $L$ simpler shapes (e.g. rectangles, ellipsoids, etc.) via $\sum_{l} \int_{\mathcal{A}_{l}} p\left(\mathbf{r}_{n} \mid \mathbf{m}_{0: n}\right) d \mathbf{r}_{n}$.

Compared to examining specific spatial points or locations, e.g. in [9], [15], calculating the probability that geographical area(s) or any point/region within it/them in this paper is a more generic and fitting formulation for the tackled problem of early prediction of a threatening or anomalous drone behaviour.

\section{EXPERIMENTAL RESUlts}

To show the capability of the proposed intent inference approach real measurements, namely 3-D positional information of the tracked-recognised drone, from Aveillant's Gamekeeper radar is used below. They are for a drone flight from the SESAR SAFIR live demonstrations performed close to the Port of Antwerp, Belgium, see [24]. We specifically consider the task of predicting, as early as possible, when the drone intends to leave an authorised flying zone, if at all. The covariance of the noisy 3-D Cartesian observations from the radar is given by $\Sigma_{n}=15^{2} I_{3 \times 3}$.

\section{A. Overview of Aveillant Gamekeeper radar}

The Aveillant Gamekeeper 16U staring radar system in Figure 1 has been specifically designed for high performance detection-recognition of drones within the range of $7.5 \mathrm{~km}$ in its current configuration. It differs fundamentally from existing mechanical or electronic-steering scanning radars by continuously illuminating the volume of interest on transmit and forms multiple simultaneous receive beams. This enables continuous dwelling on target and provides exceptionally detailed target specific measurements (e.g. high Doppler resolution) that are utilised by specialised processing techniques for better detection, tracking in 3-D and discrimination of slow, low altitude, and small UAS targets [2], [3].

\section{B. Considered Scenarios}

Here, a "permitted" geographical area $\mathcal{A}$ is artificially chosen to enable assessing the following two settings:

- Drone remains in the region: in this example $\mathcal{A}$ covers all of the six waypoints of the drone trajectory as depicted in Figure 2. It can be seen from the figure that the calculated probability of the drone intending to remain in the authorised area (or equivalently having a destination within it) at each time step is noticeably high. For instance, the introduced prediction algorithm reports probabilities consistently above 0.8 when $\sigma_{r}=25$. Although $\sigma_{r}=0$ consistently produces probabilities of one, the model in this case assumes a fixed latent destination which renders the proposed approach not capable of effectively handling change in intent (e.g. exist or re-entry as in the next

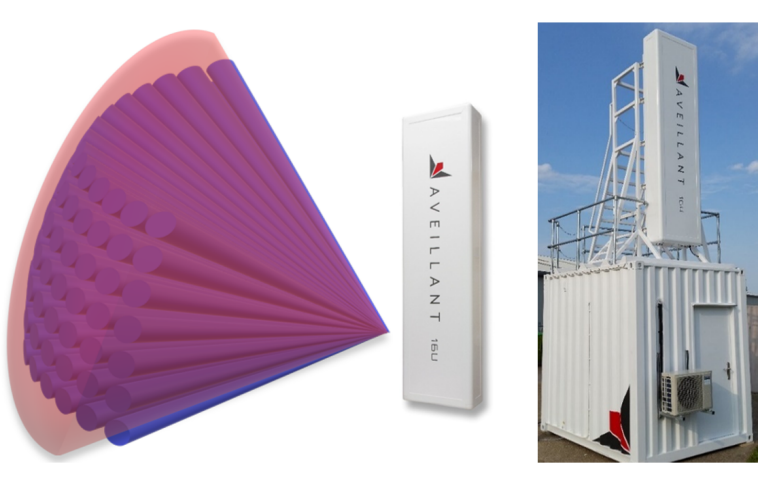

Fig. 1. Aveillant's Gamekeeper staring radar. 

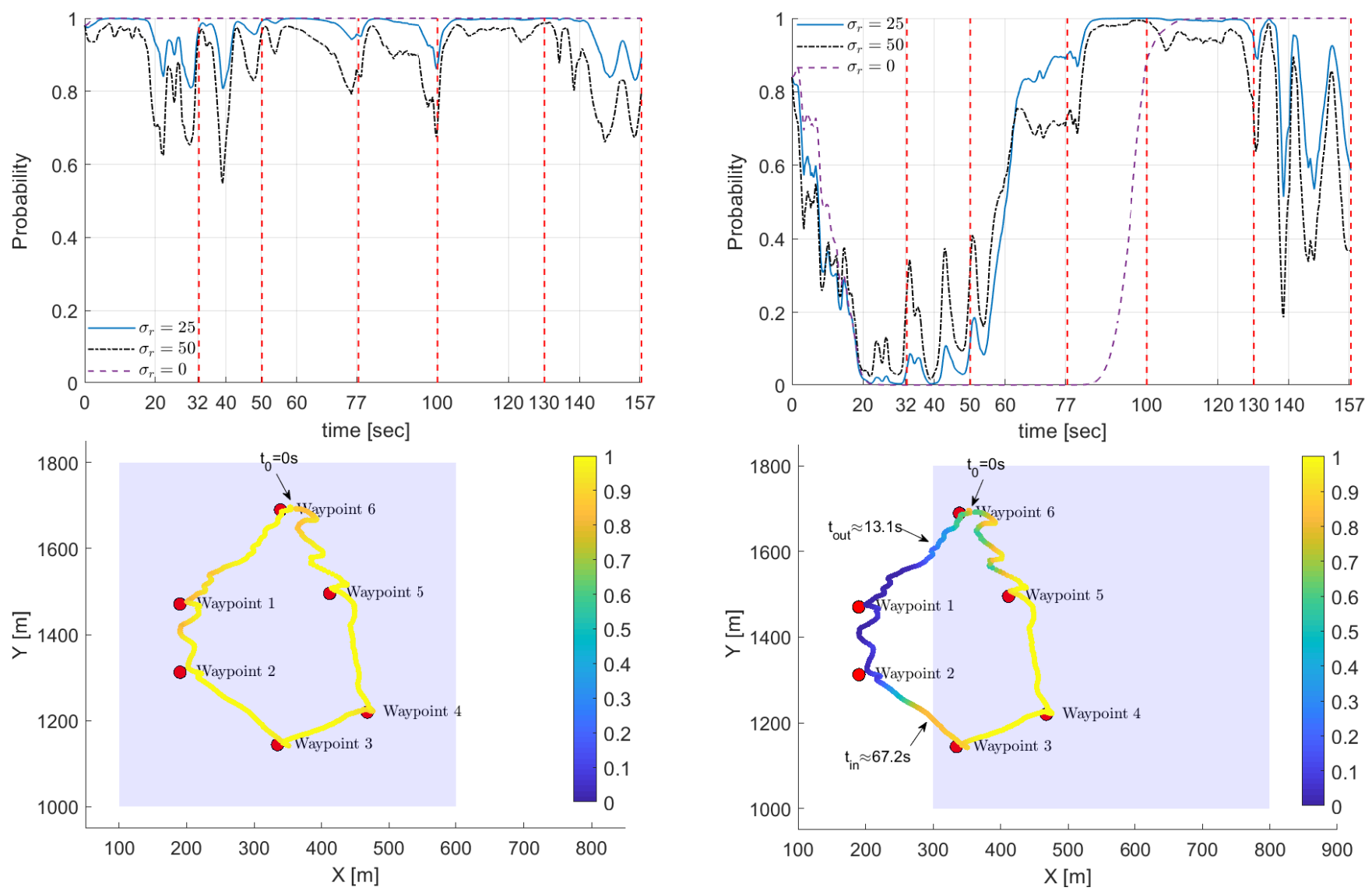

Fig. 2. A drone flying in a permitted zone $\mathcal{A}$ (shaded region). Top: estimated probabilities of the drone intending to stay in $\mathcal{A}$ for various parameters of the proposed approach; vertical lines indicate the timings when the UAS reaches each of its six waypoints. Bottom: filtered target track and its color signifies the probability of UAS remaining in $\mathcal{A}$ for $\sigma_{r}=25 ; t_{0}=0$ s is the flight start time.

example). Further, several fluctuations in the calculated probabilities can be observed. This is to be expected and reflects the fact that the algorithm does respond to a target behaviour that might suggest it is intending to exist $\mathcal{A}$ such as approaching the region boundary (e.g. whilst heading to waypoint 6 towards the end of the flight) or seemingly heading there (e.g. when it nears waypoint 1 and travels between waypoints 1 and 2). At such time instants, the drone velocity can be key to determine the underlying intent; this is seamlessly considered by the proposed inference technique and it is incorporated in the adopted extended motion and intent model.

- Drone exits and re-enters the region: in Figure 3 the authorised zone $\mathcal{A}$ is now shifted such that the drone waypoints 1 and 2 are outside $\mathcal{A}$. It is clear from the figure that the applied predictor can reveal, away from the boundary and with increased confidence, the drone intent to: a) leave $\mathcal{A}$ shortly after take-off at $t_{0}$ (i.e. near waypoint 6 ) where the probability rapidly decreases during this flight section and then reduces to nearly zero as the target exits the permitted region, and $b$ ) enter again $\mathcal{A}$ when travelling from waypoint 2 to 3 and quickly

Fig. 3. An example with the drone exiting region $\mathcal{A}$ (shaded region) at $t_{\text {out }} \approx 13.1 \mathrm{~s}$ and then re-enters at $t_{\text {in }} \approx 67.2 \mathrm{~s} ; t_{0}=0 \mathrm{~s}$ is the flight start time. Top: estimated probability of the target intent to remain in $\mathcal{A}$ and the six vertical lines indicate when the drone reached each of the six waypoints in its trajectory. Bottom: filtered target track and its color signifies probability of the drone remaining in or entering $\mathcal{A}$ for $\sigma_{r}=25$.

becomes nearly 1 as the UAS re-enters the authorised area. Conversely, region $\mathcal{A}$ can be treated as a prohibited region and the objective is then to detect if the drone aims to reach it. In this case, the predictor can infer the UAS intent to enter this region well before it does (approximately $17.2 \mathrm{~s}$ in advance from waypoint 2). It can also be noticed that there are several drops (except when $\sigma_{r} \neq 0$ ) in probabilities when the drone travelled from waypoint 5 to 6 . This is attributed to the drone undertaking manoeuvring turns during this leg of the journey and its velocity can support the possibility that it might leave the area. The algorithm responds accordingly, especially as the drone nears the boundary of $\mathcal{A}$ at waypoint 6 . It is noted that such unsmooth track sections can be induced by noisy sensor observations. Again, assuming a fixed latent destination makes the algorithm insensitive to the change of intent; this is obvious from Figure 3 top panel where the predictor with zero destination process noise takes substantially longer time to react to the re-entry intent in comparison to a non-zero $\sigma_{r}$ accounting for a dynamically changing intent.

It must be noted that the available short real drone trajectory 
(just over two minutes) is for a benign waypoint driven flight. This should be taken into account when assessing the advantages of the presented inference framework, namely how early it can reveal a malicious behaviour should be viewed relative to the corresponding time between a waypoint and the drone exist/entry location to $\mathcal{A}$. Whilst remarkably early intent predictions can be delivered by the introduced algorithms for longer UAS threatening tracks, this will require further evaluation from representative data for such scenarios; a topic that can be addressed in future work.

The above two examples clearly illustrate the efficacy of the proposed approach, which can give additional time for the non-cooperative surveillance system to take timely action to minimise the impact of a drone threatening or anomalous behaviour. This includes warning the drone operator (if possible) and employing secondary systems. On the other hand, alternative simple methods (e.g. those based on the observed drone's positions and proximity to a region) do not have a predictive capability and typically report a problem when the drone has already left (or reached) $\mathcal{A}$. They can also be prone to errors due to inaccurate sensor observations, unlike here where suitable models are applied and inference is done sequentially such that the intent prediction certainty is propagated throughout the target track. Additionally, late threat detection can in some cases lead to drastic measures such as triggering an alarm and closure of airspace.

\section{CONCLUSiON}

A Bayesian inference approach for the detection of drone threatening behaviour in a surveyed region is presented. Whilst it is discussed in the context of radar-based non-cooperative drone surveillance, the introduced framework is agnostic to the employed sensor (i.e. not limited to radar) and/or the target type (i.e. not necessarily a UAS). The intent detection approach can facilitate automated decision making and resource allocation in UMTS. More importantly, it is softwarebased and can be employed by an extant systems, e.g. at the command and control level. In a future publication, we will report on additional work that we have carried out under this DSTL contract in which waypoints for drones are explicitly detected and learned from the data within a jump particle filter framework, reporting tracking as well as more intent prediction results.

\section{ACKNOWLEDGMENT}

Authors thank Aveillant for providing real radar data and supporting this work. We acknowledge DSTL's financial support via DASA (Countering Drones Phase 1) under contract DSTLX1000144447.

\section{REFERENCES}

[1] SESAR JU, "Single European sky ATM research joint undertaking," http://www.sesarju.eu/, Accessed: 26/04/2021.

[2] M. Jahangir and C. J. Baker, "L-band staring radar performance against micro-drones," in 2018 19th International Radar Symposium (IRS). IEEE, 2018, pp. 1-10.
[3] M. Jahangir and J. Baker, "Characterisation of low observable targets with a multi-beam staring radar," in International Conference on Radar Systems (Radar 2017). 2017, IET.

[4] S. Harman, "Analysis of the radar return of micro-UAVs in flight," in 2017 IEEE Radar Conference (RadarConf). IEEE, 2017, pp. 1159-1164.

[5] Civil Aviation Authority, "Airspace restrictions for unmanned aircraft and drones," https://www.caa.co.uk/Consumers/Unmanned-aircraft/Ourrole/Airspace-restrictions-for-unmanned-aircraft-and-drones, Accessed: 26/04/2021.

[6] K. Saleh, M. Hossny, and S. Nahavandi, "Intent prediction of pedestrians via motion trajectories using stacked recurrent neural networks," IEEE Transactions on Intelligent Vehicles, vol. 3, no. 4, pp. 414-424, 2018.

[7] B. Völz, H. Mielenz, I. Gilitschenski, R. Siegwart, and J. Nieto, "Inferring pedestrian motions at urban crosswalks," IEEE Transactions on Intelligent Transportation Systems, 2018.

[8] S. Gaurav and B. D. Ziebart, "Discriminatively learning inverse optimal control models for predicting human intentions," in International Conference on Autonomous Agents and Multiagent Systems, 2019.

[9] B. I. Ahmad, J. K. Murphy, P. M. Langdon, S. J. Godsill, R. Hardy, and L. Skrypchuk, "Intent inference for hand pointing gesture-based interactions in vehicles," IEEE Transactions on Cybernetics, vol. 46, no. 4, pp. 878-889, 2016.

[10] B. I. Ahmad, J. K. Murphy, P. M. Langdon, and S. J. Godsill, "Bayesian intent prediction in object tracking using bridging distributions," IEEE Transactions on Cybernetics, vol. 48, no. 1, pp. 215-227, 2018.

[11] J. Liang, B. I. Ahmad, R. Gan, P. Langdon, R. Hardy, and S. Godsill, "On destination prediction based on Markov bridging distributions," IEEE Signal Processing Letters, vol. 26, no. 11, pp. 1663-1667, 2019.

[12] J. Liang, B. I. Ahmad, and S. Godsill, "Simultaneous intent prediction and state estimation using an intent-driven intrinsic coordinate model," in 2020 IEEE 30th International Workshop on Machine Learning for Signal Processing (MLSP), 2020, pp. 1-6.

[13] L. Vladimirov and S. Maskell, "A SMC sampler for joint tracking and destination estimation from noisy data," in 2020 IEEE 23rd International Conference on Information Fusion (FUSION), 2020, pp. 1-8.

[14] B. I. Ahmad, J. K. Murphy, S. Godsill, P. Langdon, and R. Hardy, "Intelligent interactive displays in vehicles with intent prediction: A Bayesian framework," IEEE Signal Processing Magazine, vol. 34, no. 2, pp. 82-94, 2017.

[15] R. Gan, J. Liang, B. I. Ahmad, and S. Godsill, "Modeling intent and destination prediction within a Bayesian framework: Predictive touch as a usecase," Data-Centric Engineering, vol. 1, pp. e12, 2020.

[16] R. Rezaie and X. R. Li, "Destination-directed trajectory modeling, filtering, and prediction using conditionally markov sequences," IEEE Transactions on Aerospace and Electronic Systems, 2020.

[17] L. M. Millefiori, P. Braca, K. Bryan, and P. Willett, "Modeling vessel kinematics using a stochastic mean-reverting process for long-term prediction," IEEE Transactions on Aerospace and Electronic Systems, vol. 52, no. 5, pp. 2313-2330, 2016

[18] M. Üney, L. M. Millefiori, and P. Braca, "Data driven vessel trajectory forecasting using stochastic generative models," in 2019 IEEE International Conference on Acoustics, Speech and Signal Processing (ICASSP). IEEE, 2019, pp. 8459-8463.

[19] R. P. S. Mahler, Statistical Multisource-Multitarget Information Fusion, Artech House, Inc., USA, 2007.

[20] S. K. Pang, J. Li, and S. J. Godsill, "Detection and tracking of coordinated groups," IEEE Transactions on Aerospace and Electronic Systems, vol. 47, no. 1, pp. 472-502, January 2011.

[21] D. J. Salmond and N. J. Gordon, "Group and extended object tracking," in Signal and Data Processing of Small Targets 1999, O. E. Drummond, Ed. International Society for Optics and Photonics, 1999, vol. 3809, pp. 284 - 296, SPIE

[22] G. Zhou, K. Li, and T. Kirubarajan, "Constrained state estimation using noisy destination information," Signal Processing, vol. 166, pp. 107226, 2020.

[23] R. Gan, B. I. Ahmad, and S. Godsill, "Lévy state-space models for tracking and intent prediction of highly manoeuvrable objects," IEEE Transactions on Aerospace and Electronic Systems, 2021, Accepted.

[24] Project SAFIR, "Safe and flexible integration of initial U-space services in a real environment," https://www.sesarju.eu/index.php/projects/safir, Accessed: 26/04/2021. 